\title{
Diferencia esperada de progenie para peso al destete en selección de vaquillas mestizas en Manabí
}

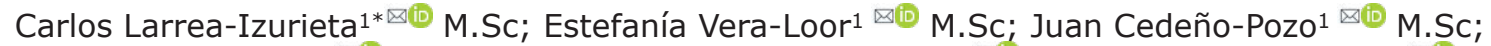 \\ Roberto Maingón-Navia2 ${ }^{凶}$ MVZ; Limberg Zambrano-Pinargote ${ }^{3} \bowtie \mathbb{C}^{-}$M.Sc; Luis Condo-Plaza4 ${ }^{凶}$ M.Sc. \\ ${ }^{1}$ Escuela Superior Politécnica Agropecuaria de Manabí "Manuel Félix López", Carrera de Medicina Veterinaria, Campus Politécnico El \\ Limón, Km 2.7 vía Calceta-El Morro-El Limón, sector El Gramal. Calceta, Manabí, Ecuador. \\ ${ }^{2}$ Agrícola El Naranjo, Hacienda Napo, San Vicente, $\mathrm{Km} 5$ vía a Canoa, San Vicente, Manabí, Ecuador. \\ 3Universidad Laica "Eloy Alfaro" de Manabí, Extensión Chone, Facultad de Ciencias Agropecuarias, Carrera de Ingeniería Agropecuaria, \\ Av. Eloy Alfaro, Chone, Manabí, Ecuador \\ ${ }^{4}$ Escuela Superior Politécnica de Chimborazo, Facultad de Ciencias, Escuela de Matemáticas y Estadística, Km 1.5 Panamericana Sur, \\ Riobamba, Chimborazo, Ecuador. \\ *Correspondencia: colirbba@hotmail.com
}

Recibido: Agosto 2018; Aceptado: Noviembre 2018; Publicado: Abril 2019.

\section{RESUMEN}

Objetivo. Determinar la diferencia esperada de la progenie (DEP) para el peso al destete ajustado a 205 días (PD) en hembras Brahman mestizas nacidas en el año 2016 como criterio de selección de futuras reproductoras. Materiales y métodos. Se analizaron 3467 registros generados entre 1983 y 2016 en la hacienda Napo (San Vicente, Manabí, Ecuador). El modelo estadístico y animal incluyó el efecto aleatorio del padre y como efectos fijos: composición racial, sexo y año de nacimiento. El análisis de la varianza fue realizado mediante el procedimiento GLM del paquete estadístico SAS. Los componentes de la varianza entre y dentro de padre para calcular la heredabilidad ( $h^{2}$ ) del PD y los valores de cría, se utilizó el software MTDFRELM, a través del sistema de evaluación del Mejor Predictor Lineal Insesgado (BLUP).

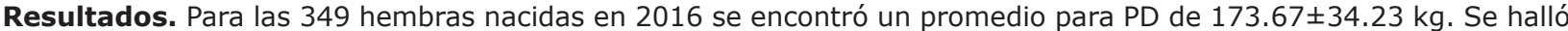
diferencia altamente significativa $(p \leq 0.01)$ para los efectos aleatorios y fijos, $h^{2}$ para PD de $0.13 \pm 0.04$ y las DEP variaron entre -6.13 y $+5.58 \mathrm{~kg}$ con rango entre los valores de cría de $11.71 \mathrm{~kg}$ y una exactitud entre 0.50 y 0.72 . Conclusión. La baja $h^{2}$ encontrada para PD manifiesta la alta influencia de los factores no genéticos, sin embargo las hembras con mayor valor DEP pueden mejorar el desempeño en este parámetro; se hace necesario identificar el mejor cruce racial que se adapte a la explotación con el fin de obtener crías con alto rendimiento en el PD.

Palabras clave: Bovinos, cruzamiento, heredabilidad, valor genético (Fuente: Agrovoc).

\begin{abstract}
Objective. Determine the progeny difference expected (DEP) for the weaning weight adjusted to 205 days (PD) in crossbreed Brahman females born in 2016 as selection criteria for future breeders. Materials and methods. It is analyzed 3467 records generated between 1983 and 2016 in the Napo farm (San Vicente, Manabí, Ecuador). The statistical model included the random effect of the father and as fixed effects: breed composition, sex and year of birth. The variance analysis was performed using the GLM procedure of the SAS statistical package. The components of the variance between and within the father to calculate the heritability $\left(h^{2}\right)$ of the PD and the breeding values, the MTDFRELM software was used, applying the animal model, through the evaluation system of the Best Linear Unbiased Predictor (BLUP). Results. It was found that the PD average for the 349 females born in 2016 was $173.67 \pm 34.23 \mathrm{~kg}$. A highly significant difference ( $p \leq 0.01$ ) was found for the random and fixed effects. The $h^{2}$ for PD was $0.13 \pm 0.04$. The DEP fluctuated between -6.13 and $+5.58 \mathrm{~kg}$ with a range between the breeding values of $11.71 \mathrm{~kg}$ and an accuracy between 0.50 and 0.72 . Conclusion. The low $h^{2}$ found for PD shows the high influence of non-genetic factors; it is necessary to identify the best crossbreed that adapts to the livestock in order to obtain high performance products in the PD.
\end{abstract}

Keywords: Breeding value, cattle, crossing, heritability. (Source: Agrovoc).

Como citar (Vancouver)

Larrea-Izurieta C; Cedeño-Pozo J, Maingón-Navia R, Vera-Loor E, Zambrano-Pinargote L, Condo-Plaza L. Diferencia esperada de progenie para peso al destete en selección de vaquillas mestizas en Manabí. Rev MVZ Cordoba. 2019; 24(2):7193-7197. DOI: https://doi.org/10.21897/rmvz.1293

(C)E (los) autor (es), Revista MVZ Córdoba 2019. Este artículo se distribuye bajo los términos de la licencia internacional Creative Commons Attribution 4.0 (https://creativecommons.org/licenses/by-sa/4.0/), que permite el uso sin restricciones, la distribución y la reproducción en cualquier medio, siempre que se otorgue el crédito apropiado al autor o autores originales y la fuente. 


\section{INTRODUCCIÓN}

El Instituto Nacional de Estadísticas y Censos (1), indica que la población de ganado vacuno en la provincia de Manabí fue de 896,479 cabezas, lo que representa el $21.39 \%$ a nivel nacional, ocupando el primer lugar en este censo. Experiencias de productores ganaderos, especialmente de la región Costa, dan cuenta de que la producción de bovinos de carne como actividad ha venido perdiendo terreno donde su competitividad y sostenibilidad se encuentran en discusión; el estado ha venido realizando desde el año 2010 algunas inversiones orientadas a la potencialización del sector ganadero de carne (2).

La producción de bovinos de carne en la mayoría de las explotaciones en el trópico ecuatoriano, se caracteriza por el limitado o al poco uso de registros genealógicos, productivos y reproductivos, lo que condiciona la estimación de los parámetros genéticos, restringiendo el avance genético de las explotaciones; generalmente se realiza una distinción visual a los animales en forma individual o grupal, seleccionando los ejemplares que formarán parte del rebaño, brindando muy poca orientación ya que se selecciona el animal por fenotipo más no por genotipo. Con lo indicado, se hace necesaria la utilización de algunos modelos matemáticos, que permiten separar los efectos genéticos de los no genéticos para realizar la valoración, la misma que se puede estimar a través de la información del mismo individuo, de sus parientes y/o hijos (3).

La diferencia esperada de la progenie (DEP), es la predicción genética que se pueden estimar para cualquier característica animal, que a su vez, pueda ser medida con precisión, también son consideradas como la desviación de un valor base determinado para cada raza y con ello es posible la predicción del valor genético de cada individuo (mérito genético) (4), dado que las diferencias en las DEP permite incorporar al hato animales seleccionados por su alto valor genético para las características económicas productivas que se desean mejorar en función de las decisiones que considere cada criador según los objetivos de producción.

Se contempló como objetivo predecir las DEP para el peso al destete ajustado a 205 días (PD) de las hembras mestizas nacidas en el año 2016 y utilizar esta información como criterio de selección de las mismas.

\section{MATERIALES Y MÉTODOS}

Ubicación. La información analizada se recabó en la hacienda Napo, adjunta a la empresa "Agrícola El Naranjo", ubicada en el cantón San Vicente, (ManabíEcuador).

Sitio experimental. Presenta características agroecológicas de bosque premontano seco tropical, con una temperatura media anual de $27^{\circ} \mathrm{C}$ e intensidad de lluvias de $700 \mathrm{~mm}$ anuales. La explotación tiene una extensión de 1019 hectáreas, de las cuales, el 54\% se dedica a la ganadería con pastizales compuestos principalmente de Panicum maximum, que se rotan cada 8 días; la topografía del área productiva está distribuida el $22 \%$ en potreros planos y el $78 \%$ en lomas suaves con una inclinación de 30\% aproximadamente.
Animales. Se analizaron 3467 registros de peso al destete entre los años 1983 y 2016, provenientes de las fichas individuales de los animales que estaban ingresados en el software InterHerd (5), implementado en la explotación en 1999. La mayor parte de estos registros no contaba con la información del padre, considerando esta limitante se analizaron los 30 machos reproductores que constaban en los registros analizados, los mismos que transmitieron su potencial genético a través de la inseminación artificial, el resto fue producto de inseminaciones o monta directa con toros puros y mestizos que no constaban en los registros.

Se encontraron 266 composiciones raciales, con base Brahman (BR), cruzadas con Senepol (SE), Angus Rojo $(A R)$, Nellore (NE), Rojo Sueco (RS), Pardo Suizo (PS), Gyr (GY), Holstein Friesian (HO), Jersey (JE), Charolais $(\mathrm{CH})$, Piamontesa (PM), Chianina (CI), Limousin (LM) y Simmental (SI), todas en diferentes proporciones entre dos y cinco razas distintas por animal. Las particularidades obtenidas se examinaron en una base de datos creada en Microsoft Excel 2016. Los pesos al destete fueron ajustados a 205 días, utilizando la fórmula sugerida por Silva et al (6) en Charolais.

Análisis estadístico. El análisis de la varianza para los efectos fijos y aleatorios que intervienen en el PD, fue realizado mediante el procedimiento GLM del paquete estadístico SAS v9.4 (2013), en el modelo se incluyó el efecto aleatorio del toro y los efectos fijos de los grupos contemporáneos en los que se consideró la composición racial, sexo y año de nacimiento de cada uno de los individuos usando el siguiente modelo:

$Y_{i j k l m}=\mu+A_{i}+B_{j}+C_{k}+D_{1}+e_{i j k l m}$

$\mathrm{Y}_{\mathrm{ijklm}}=$ Peso ajustado de las crías a los 205 días de edad $(\mathrm{kg})$;

$\mu=$ Promedio general del peso al destete $(\mathrm{kg})$;

$A_{i}=$ Efecto del $i$-ésimo toro (30 toros), $i=1$...30;

$\mathrm{B}_{j}=$ Efecto de la j-ésima composición racial (266 proporciones raciales con base brahman), $j=1 \ldots 266$;

$\mathrm{C}_{\mathrm{k}}=$ Efecto del k-ésimo sexo de la cría (hembra, macho), $\mathrm{k}=1,2$;

$D_{1}=$ Efecto del I-ésimo año de nacimiento (de 1983 a 2016) $\mid=1 \ldots 34$;

$\mathrm{e}_{\mathrm{ijklm}}=$ Efecto residual.

Estimación de la DEP. Para estimar la heredabilidad $\left(h^{2}\right)$ del PD y los valores de cría de la población para el mencionado carácter de las 349 hembras que nacieron en el año 2016, se corrieron los datos en el software MTDFRELM aplicando el modelo animal, considerando los grupos contemporáneos en función de la composición racial, sexo y año de nacimiento de cada uno de los individuos como efecto fijo y como efecto aleatorio los efectos genéticos aditivos a través del sistema de evaluación del Mejor Predictor Lineal Insesgado (BLUP) (7). El modelo fue el siguiente:

$\mathrm{Y}=\mathrm{Xb}+\mathrm{Za}+\mathrm{e}$

$\mathrm{Y}=$ Vector de los registros de peso ajustado de las crías a los 205 días de edad (kg);

$\mathrm{b}=$ Vector de los efectos fijos incluyendo los grupos contemporáneos definidos por la combinación de la composición racial, sexo y año de nacimiento;

$a=$ Vector de los efectos aleatorios genéticos aditivos directos;

$\mathrm{X}, \mathrm{Z}=$ Matrices de incidencia que relacionan las observaciones en $Y$ con los respectivos efectos; $\mathrm{e}=$ Vector de residuales aleatorios. 
Una vez hallados los valores de las DEP, se listaron las hembras nacidas en el año 2016 con mayor valor en las DEP, las mismas que servirán para formar el grupo genético de élite.

\section{RESULTADOS}

Desempeño. En el análisis de la varianza para el PD se encontró diferencias altamente significativas $(p \leq 0.01)$ en todas las fuentes de variación (padre, raza, sexo y año de nacimiento), como se puede apreciar en la tabla 1 . El promedio total histórico entre 1983 y 2016 para el PD fue de $149.16 \pm 26.70 \mathrm{~kg}$ y para las 349 hembras nacidas en el año 2016 fue de $173.67 \pm 34.23 \mathrm{~kg}$, superando ampliamente estas últimas en $24.51 \mathrm{~kg}$ al promedio total.

Tabla 1. Análisis de la varianza para el peso al destete ajustado a 205 días.

\begin{tabular}{ccccc}
\hline $\begin{array}{c}\text { Fuente de } \\
\text { variación }\end{array}$ & $\begin{array}{c}\text { Grados de } \\
\text { libertad }\end{array}$ & $\begin{array}{c}\text { Cuadrados } \\
\text { medios }\end{array}$ & Valor F & p-valor \\
\hline Padre & 29 & 2193.61 & 4.50 & $<0.001 * *$ \\
Raza & 265 & 1348.98 & 2.77 & $<0.001 * *$ \\
Sexo & 1 & 135911.07 & 278.91 & $<0.001 * *$ \\
$\begin{array}{c}\text { Año de } \\
\text { nacimiento }\end{array}$ & 33 & 11649.44 & 23.91 & $<0.001 * *$ \\
Error & 3466 & 2470543.90 & & \\
\hline
\end{tabular}

** Diferencia altamente significativa $(p \leq 0.01)$

DEP por animal. La $\mathrm{h}^{2}$ para la mencionada característica en esta investigación fue de $0.13 \pm 0.04$. Las frecuencias absolutas y relativas de las DEP para los animales evaluados en el año 2016, el $41.49 \%$ de las hembras nacidas en ese año, presentó un valor de cría entre +0.38 y $+5.58 \mathrm{~kg}$ variando entre 0.52 y 0.72 de exactitud con un promedio de 0.70 y el restante $58.51 \%$ presentó valores genéticos entre -6.13 y $+0.38 \mathrm{~kg}$ variando entre 0.50 y 0.72 con un promedio de 0.69 de exactitud en los valores de DEP (Tabla 2).

Tabla 2. Frecuencia de las diferencias esperadas de la progenie de los animales analizados.

\begin{tabular}{cccccc}
\hline Clase & $\begin{array}{c}\text { Límite } \\
\text { inferior }\end{array}$ & $\begin{array}{c}\text { Límite } \\
\text { superior }\end{array}$ & $\begin{array}{c}\text { Marca de } \\
\text { clase }\end{array}$ & $\begin{array}{c}\text { *Frecuencia } \\
\text { absoluta } \\
(\mathbf{N})\end{array}$ & $\begin{array}{c}\text { Frecuencia } \\
\text { relativa } \\
(\%)\end{array}$ \\
\hline Muy alto & +4.28 & +5.58 & 4.93 & 4 & $1.15 \%$ \\
Alto & +2.98 & +4.28 & 3.63 & 14 & $4.03 \%$ \\
$\begin{array}{c}\text { Medio alto } \\
\text { Ligeramente }\end{array}$ & +1.68 & +2.98 & 2.33 & 37 & $10.66 \%$ \\
$\begin{array}{c}\text { alto } \\
\text { Incierto }\end{array}$ & +0.38 & +1.68 & 1.03 & 89 & $25.65 \%$ \\
Ligeramente & -2.23 & -0.92 & -1.57 & 53 & $15.27 \%$ \\
$\begin{array}{c}\text { bajo } \\
\text { Medio bajo }\end{array}$ & -3.53 & -2.23 & -2.88 & 29 & $8.36 \%$ \\
Bajo & -4.83 & -3.53 & -4.18 & 10 & $2.88 \%$ \\
Muy bajo & -6.13 & -4.83 & -5.48 & 2 & $0.58 \%$ \\
\hline
\end{tabular}

*Número de hembras nacidas en el año 2016.

La mayor frecuencia (31.41\%) corresponde a los animales con valores de cría entre -0.92 y $+0.38 \mathrm{~kg}$. Al considerar también los valores positivos de la clase incierta de $D E P$, se redistribuyeron las proporciones considerando los valores de cría desde cero, obteniéndose que el
$51.59 \%$ de las hembras tienen valores de cría positivos y el $48.41 \%$ presentó valores negativos, manteniendo los mismos promedios y valores máximos y mínimos sin redistribuir la clase incierta.

DEP por mestizaje. De acuerdo con los valores de cría para los 12 mestizajes más frecuentes de las 349 hembras nacidas en 2016, se observó que el cruce $50 \%$ $B R \times 50 \% A R$, que representa el $5.44 \%$ de la población de hembras en estudio y presentó el mayor valor genético $(+2.70 \mathrm{~kg})$; mientras que el mestizaje $50 \%$ BR $\times 25 \%$ SE $\times 25 \%$ AR, que es el más frecuente (15.47\%) contó con un valor genético de $-2.11 \mathrm{~kg}$, siendo este último superior al de la raza BR que manifestó un valor de cría promedio de -3.45 y frecuentó en un $9.74 \%$ de las hembras analizadas, como se muestra en la tabla 3.

Tabla 3. Valor de cría para las 12 composiciones raciales más frecuentes de la población analizada.

\begin{tabular}{cccc}
\hline Composición racial & N & Frecuencia & DEP \\
\hline $\operatorname{BR}(50 \%) \times \operatorname{SE}(25 \%) \times A R(25 \%)$ & 54 & $15.47 \%$ & -2.11 \\
$\operatorname{BR}(100 \%)$ & 34 & $9.74 \%$ & -3.45 \\
$\operatorname{SE}(50 \%) \times B R(25 \%) \times A R(25 \%)$ & 23 & $6.59 \%$ & -0.50 \\
$\operatorname{SE}(63 \%) \times B R(25 \%) \times A R(13 \%)$ & 22 & $6.30 \%$ & -1.80 \\
$\operatorname{BR}(50 \%) \times A R(50 \%)$ & 19 & $5.44 \%$ & +2.70 \\
$\operatorname{BR}(50 \%) \times \operatorname{SE}(50 \%)$ & 18 & $5.16 \%$ & -1.12 \\
$\operatorname{SE}(38 \%) \times A R(38 \%) \times B R(25 \%)$ & 13 & $3.72 \%$ & -3.19 \\
$\operatorname{BR}(50 \%) \times N E(50 \%)$ & 13 & $3.72 \%$ & -1.40 \\
$\operatorname{SE}(75 \%) \times B R(25 \%)$ & 9 & $2.58 \%$ & +0.36 \\
$\operatorname{BR}(50 \%) \times R S(50 \%)$ & 8 & $2.29 \%$ & +0.04 \\
$\operatorname{BR}(50 \%) \times A R(38 \%) \times \operatorname{SE}(12 \%)$ & 8 & $2.29 \%$ & -2.56 \\
$\operatorname{SE}(75 \%) \times B R(13 \%) \times A R(13 \%)$ & 7 & $2.01 \%$ & -9.44 \\
\hline
\end{tabular}

$\mathrm{BR}=$ Brahman; $\mathrm{SE}=$ Senepol; $\mathrm{AR}$ = Angus rojo; NE = Nellore; RS = Sueco rojo.

También se encontró en esta investigación que el cruce $25 \%$ BR x 25\% SE x 25\% AR x 25\% HO, presentó el valor de cría más alto $(+17.35 \mathrm{~kg})$ y el mestizaje $81 \%$ SE $x$ $13 \%$ AR x 6\% BR alcanzó el mérito más bajo $(-34.76 \mathrm{~kg})$. Cada una de estas proporciones raciales, correspondía al $0.29 \%$ de la población de hembras nacidas en el año 2016 y consideradas como los cruces menos frecuentes.

DEP por año de nacimiento. Se observó una tendencia positiva en el transcurso de los últimos 3 años para el incremento del mérito genético llegando a $+18.86 \mathrm{~kg}$ y el promedio para el PD alcanzó a $173.67 \mathrm{~kg}$ en el año 2016 (Figura 1).

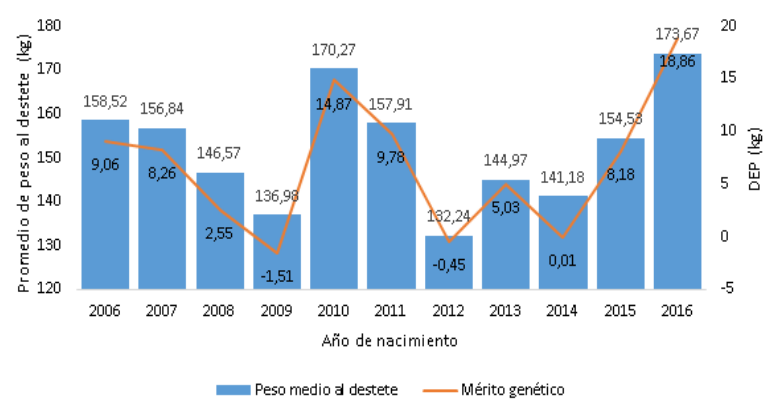

Figura 1. Promedio de peso al destete ajustado a 205 días y mérito genético de los últimos 10 años. 


\section{DISCUSIÓN}

EI PD hallado en esta investigación es superior a los reportados por Manzi et al (8) en Ruanda e inferior a los indicados por Vega et al (9) en México y Regatieri et al (10) en Brasil; el promedio obtenido en el PD, se vio afectado por una serie de factores no genéticos, entre los cuales se destaca el sistema de manejo implementado en la explotación para alimentar a los animales en períodos de sequía y por factores genéticos como son la selección de toros, vacas y vaquillas, estas dos últimas en función de su habilidad materna.

El cálculo de la $h^{2}$ es de gran importancia en el valor genético de los reproductores y en la predicción de la respuesta de selección (11); la heredabilidad es un parámetro genético propio de una población dada en un momento determinado, lo que quiere decir que esta varía de población en población, y es fundamental para la definición de los métodos de selección y además estima la relación entre el genotipo y el fenotipo (12). El valor estimado de la $\mathrm{h}^{2}$ para PD de esta población en estudio, es superior a los valores reportados por Espinoza et al (13) en ganado Cebú y Ríos et al (14) en ganado Indubrasil e inferior a los analizados por Montes et al (15), Estrada et al (16) y Crispim et al (17), en ganado Brahman y sus mestizajes.

El valor de la $h^{2}$ estimado $(0.13 \pm 0.04)$, permite indicar que existe un mayor efecto ambiental sobre esta característica en función del sistema de manejo que se aplica en la ganadería, por las condiciones de alimentación, y los criterios de selección de machos y hembras de remplazo que fueron considerados en su momento como la adaptación al medio y desempeño en el PD, a pesar de que el progreso genético será a largo plazo por el efecto del bajo valor de $h^{2}$, la misma que es afectada por las restricciones causadas por el factor ambiental propio de la explotación $(14,18)$.

En el tabla 2 se observa que las DEP para las hembras nacidas en el año 2016, oscilaron entre -6.13 y +5.58 kg correspondiendo, respectivamente, a las hembras que menor y mayor peso imprimirán en su progenie, presentando un rango de $11.76 \mathrm{~kg}$, por lo que es importante registrar los datos de los toros para completar su información productiva y reproductiva, identificando y seleccionando a los machos reproductores con DEP altamente positivos para el PD, lo que permitirá incrementar el mismo en las futuras generaciones.
En los valores de cría para los 12 mestizajes más frecuentes indicados en la tabla 3, se asume el efecto de la heterosis sobre todo el conjunto analizado, ya que el cruce entre razas puras o mestizajes diferentes dentro de esta explotación, elevan la heterosis exaltando las características productivas, los animales cruzados con genética europea tienden a mejorar estos parámetros (19), como se observó en el cruce $25 \%$ BR x $25 \%$ SE x $25 \%$ AR $\times 25 \%$ HO con una DEP de $+17.35 \mathrm{~kg}$.

Considerando los resultados que se observan en figura 1 , a pesar de tener una $h^{2}$ baja para el presente carácter, el efecto de una buena selección visual, genealógica de machos reproductores y los criterios de cruzamiento racial en los últimos tres años, fueron superiores en comparación a los siete años anteriores por lo que se debe considerar la tendencia genética, la misma que permite evaluar el progreso genético en el proceso de selección para redefinir criterios y objetivos de selección, e incrementar el cambio genético de los valores de cría predichos (20).

La baja $\mathrm{h}^{2}$ encontrada en el PD, explica una influencia muy alta de los factores no genéticos, lo que infiere en un progreso genético lento, sin embargo, se debe considerar el alto porcentaje de hembras con valores de cría positivos para formar el grupo de reproductoras élite que permitan el replanteamiento de programas de mejoramiento genético para el $\mathrm{PD}$, lo que reflejará un incremento positivo del rendimiento de este carácter dentro de la explotación, por lo que debe ser acompañado de una buena selección de machos reproductores e identificar el mejor cruce racial que se adapte a la explotación con el fin de obtener productos con alto desempeño en el PD.

\section{Conflicto de intereses}

Los autores no declaramos ningún conflicto de intereses.

\section{Agradecimientos}

A la empresa "Agrícola El Naranjo", especialmente a la Hacienda Napo, por facilitar la información para la realización de esta investigación. Los resultados del presente trabajo no reflejan la opinión o punto de vista de la empresa "Agrícola El Naranjo".

\section{REFERENCIAS}

1. INEC. Instituto Nacional de Estadísticas y Censo. Encuesta de superficie y producción agropecuaria: Ecuador: 2017. [Consultado 16 feb, 2018]. URL Valida en: http://www. ecuadorencifras.gob.ec/documentos/web-inec/ Estadisticas agropecuarias/espac/espac_2017/ Informe Ejecutivo ESPAC 2017.pdf

2. ESPOL. Estudios industriales, orientación estratégica para la toma de decisiones: Industria de la ganadería de carne. Escuela Superior Politécnica del Litoral: Ecuador: 2016. [Consultado 16 feb, 2018]. URL Valida en: http://www.espae.espol.edu.ec/wpcontent/uploads/2016/12/industriaganaderia.pdf
3. Martínez CA, Manrique PC, Elzo MA. La evaluación genética de vacunos: Una percepción histórica. Rev Colomb Cienc Pecu. 2012; 25(2):293-311. http:// aprendeenlinea.udea.edu.co/revistas/index.php/ rccp/article/view/324760

4. Madrigal M, Valverde A, Murillo O, Montero W, Muñoz B. Asociación entre marcadores genéticos CAPN-1 CAST y características de crecimiento en ganado Brahman en Costa Rica. Rev Agronomía Costarricense. 2018; 42(2):29-42. https://doi. org/10.15517/rac.v42i2.33776 
5. Agricultural Information Management Systems Limited (INTERAGRI). InterHerd Software. Reading, UK: Departament of Agriculture; 1987.

6. Silva A, Martínez JC, Briones F, Castillo SP, Hernández J. Comportamiento productivo de un hato Charolais bajo condiciones de trópico seco en Tamaulipas, México. UDO Agríc. 2013; 13(1):140145. http://www.bioline.org.br/pdf?cg13017

7. Boldman, K.; Van Vleck, L. Derivative-Free Restricted Maximum Likelihood Estimation in Animal Model with a Sparse Matrix Solver. J Dairy Sci. 1991; 74(12):4337-4343. https://doi.org/10.3168/ jds.s0022-0302(91)78629-3

8. Manzi M, Junga, JO, Ebong C, Mosi, RO. Factors affecting pre and post-weaning growth of six cattle breed groups at Songa Research station in Rwanda. Livestock Res. Rural Develop. 2012; 24(4):Artículo 68. http://www.Irrd.org//rrd24/4/manz24068.htm

9. Vega VE, Ríos A, Montaño M, Martínez G. MultipeBreed genetic evaluation of growth traits in Simmental and Simbrah cattle. Trop Subtrop Agroecosyst. 2012; 15(1):403-414. http://www. revista.ccba.uady. $\mathrm{mx} / \mathrm{ojs} /$ index.php/TSA/article/ view/1410

10. Regatieri IC, Boligon AA, Baldi F, Albuquerque LG. Genetic correlations between mature cows weight and productive and reproductive traits in Nellore cattle. Genet Mol Res. 2012; 11(3):2979-2986. https://doi.org/10.4238/2012.may.10.4

11. Montes D, Vergara O, Barragán W. Diferencia esperada de la progenie como herramienta de selección para peso al destete en ganado Brahman. Rev MVZ Córdoba. 2011; 16(1):2381-2390. https://doi.org/10.21897/rmvz.297

12. Ossa G, Suarez M, Pérez J. Efectos del medio y la herencia sobre el peso al destete de terneros de la raza Romosinuano. Rev MVZ Córdoba. 2005; 10(2):673-683. https://doi.org/10.21897/ rmvz.443

13. Espinoza JL, Palacios A, Guerra D, González $D$, Ortega R, Rodríguez F. Comparación de dos modelos para la estimación de parámetros y valores genéticos del peso en ganado Cebú. Agrociencias. 2008; 42(1):29-36. http://www.colpos.mx/ agrocien/Bimestral/2008/ene-feb/art-4.pdf
14. Ríos $A$, Hernández VD, Villagómez $E$, Zárate JP, Villagómez JA. Direct and maternal genetic effects for growth traits in Indubrazil cattle. Revista Científica FCV-LUZ. 2013; 23(5):440-447. http://www. saber.ula.ve/bitstream/handle/123456789/37537/ articulo10. PDF? sequence $=1$ \&isAllowed $=y$

15. Montes D, Vergara O, Prieto E, Rodríguez A. Estimación de los parámetros genéticos para el peso al nacer y al destete en ganado bovino de la raza Brahman, Rev MVZ Córdoba. 2008; 13(1):11841191. https://doi.org/10.21897/rmvz.409

16. Estrada RJ, Magaña JG, Segura JC. Estimation of genetic parameters for preweaning growth traits of Brahman cattle in Southeastern Mexico. Trop Anim Health Prod. 2014; 46(5):771-776. https:// doi.org/10.1007/s11250-014-0563-z

17. Crispim AC, Kelly MJ, Guimarães SEF, et al. MultiTrait GWAS and New Candidate Genes Annotation for Growth Curve Parameters in Brahman Cattle. PLoS ONE. 2015; 10(10):e0139906. https://doi. org/10.1371/journal.pone.0139906

18. Morales $Y$, Guerra $D$, Suárez MA, Rodríguez $M$, Gonzalez D, Ramos F. Parámetros y tendencia genética en rasgos de crecimiento post destete de machos de la raza Santa Gertrudis. Cuban Journal of Agricultural Science 2013; 47(1):7-12. https:// www.redalyc.org/html/1930/193028545002/

19. Vergara OD, Flórez JM, Hernández MJ, Yaguna CJ, Manco C, Barrios TE, Rico J. Efectos raciales, heterosis y parámetros genéticos para peso al nacer en una población multirracial de ganado de carne en Colombia. Livestock Res. Rural Develop. 2014; 26(3):Artículo 58. http://www.Irrd.org/Irrd26/3/ verg26058.html

20. Domínguez J, Rodríguez FA, Núñez R, Ramírez $R$, Ruiz A. Parámetros genéticos y tendencias genéticas para características de comportamiento en ganaderías de lidia mexicanas. Rev Mex Cienc Pecu. 2014; 5(3):261-271. https://doi. org/10.22319/rmcp.v5i3.3970 